\title{
Substantia stans per essentiam suam: Proclus et l'auteur du De causis sur les êtres qui se constituent eux-mêmes
}

\author{
Carlos Steel \\ KU Leuven
}

Pour examiner comment l'auteur du De causis a interprété et transformé l'Elementatio theologica de Proclus, j'ai choisi ce qui est considéré comme la doctrine la plus originale de sa métaphysique, celle qui concerne les êtres qui se constituent eux-mêmes ( $\alpha$ $\theta u \pi o ́ \sigma \tau \alpha \tau \alpha$; Éléments de théologie $§ 40-41$ ). Comme le démontre Proclus, dans la procession des êtres à partir du premier Principe, les substances incorporelles, tels l'intellect et l'âme rationnelle, ne sont pas simplement le produit de l'acte créateur de la première cause. Elles ne proviennent pas d'une cause extérieure à elles-mêmes, comme c'est le cas des êtres corporels, mais sont elles-mêmes causes de leur propre être tout en dépendant de la première cause. Pour cette raison, elles ne sont pas soumises à la génération et la corruption, mais elles jouissent d' une existence nécessaire et sempiternelle. La doctrine des $\alpha \dot{\theta} \theta \tau$ đó $\sigma \alpha \tau \alpha$ permet à Proclus de sauvegarder à la fois la transcendance de la cause première par rapport à ce qui en dérive premièrement et d' exalter la dignité et l'autonomie des substances intellectives'. J' ai choisi ce thème, parce que, depuis Thomas d'Aquin, il est souvent dit dans la littérature que l' auteur du De causis a modifié les doctrines qu' il a reprises de Proclus dans un sens créationniste et monothéiste. Dans cette optique, la doctrine des êtres auto-constituants devait poser un grand problème à l' auteur arabe qui aurait ainsi été tenté de l'adapter voire de l'éliminer. Cependant, comme je le démonterai, ce n' est pas le cas. Non seulement l' auteur a repris la doctrine de Proclus sans modifications importantes, mais encore, il lui a, pour ainsi dire, donné plus d'ampleur. Cependant, l' ambiguïté de la traduction latine a laissé les commentateurs médiévaux penser que l'auteur du $D e$ causis est intervenu dans le texte pour rendre la doctrine acceptable aux yeux d'un croyant.

1 Sur la doctrine auto-constituants chez Proclus, cf. Gersh 1973, p. 128-138; Whittaker 1984; Trouillard 1976, p. 307-321; Beierwaltes 2001, p. 123-159; Steel 1998, p. 161-175 et Steel 2006; Chlup 2012, p. 64-75. 
Chez Proclus, les propositions qui concernent les auto-constituants ( $\$ 40-51)$ se trouvent dans la première partie de l'Elementatio theologica, après les propositions qui expriment les principes généraux qui gouvernent la procession et le retour (conversion) des êtres. En effet, les $\alpha \dot{\theta} \theta v \pi \dot{\sigma} \sigma \tau \alpha \tau \alpha$ se distinguent par leur mode particulier de procession. Ils ne procèdent pas comme s'ils étaient simplement les effets d'une cause efficiente qui leur serait extérieure, mais ils se donnent eux-mêmes leur être. Dans le De causis les propositions sur les autoconstituants se trouvent dans la toute dernière partie du traité $(\operatorname{xxIV}(\mathrm{xxv})-$ $\mathrm{XXIX}(\mathrm{XXX}))^{2}$. Ces propositions sont introduites par la proposition XXIII(XXIV) qui donne la perspective dans laquelle il faut les comprendre. Ayant démontré que la première cause régit tous les êtres créés sans qu' elle se mêle à eux et perde sa transcendance, l' auteur du De causis veut dans cette dernière partie démontrer comment les êtres dérivent de la cause première dans toute leur diversité. Cette diversité ne vient pas en tant que telle de la cause première. En effet, cette cause existe dans tous les êtres «selon une disposition unique et égale pour tous», mais les êtres créés ne peuvent recevoir l'influx à partir de la cause première que «selon la mesure de leur pouvoir (secundum modum suae potentiae)», «selon la mesure de leur être propre (secundum modum esse sui)».

Parmi les choses, certaines reçoivent la cause première par une réception unitaire, d' autres la reçoivent par une réception multipliée; certaines par une réception éternelle, $d$ ' autres par une réception temporelle; certaines par une réception spirituelle, d'autres par une réception corporelle.

Cette proposition XXIII(XXIV) est une adaptation judicieuse dans un sens monothéiste de Proclus, Éléments de théologie §142. Pour Proclus, il s'agit d'expliquer comment «les dieux» sont présents dans les êtres.

Tandis que les dieux sont présents à tous les êtres de la même manière, tous les êtres ne sont pas présents de la même manière aux dieux, mais chacun, selon son rang et sa puissance, prend sa part de la présence des

2 Comme je ne connais pas l' arabe, je citerai le Liber de causis dans la traduction latine (avec la numérotation des propositions de la tradition latine). Pour des passages difficiles, j' ai toujours comparé la version latine avec la traduction de l' arabe faite par Bardenhewer 1882 (reprint Frankfurt 1961) et avec la version anglaise dans Guagliardo, Hess, Taylor 1996. Pour le texte latin j'utilise l'édition de Pattin 1966 avec les corrections de Taylor 1989 et l'édition avec traduction allemande et annotations de Schönfeld 2003. Pour la traduction française du De causis j' ai cité en l' adaptant Magnard, Boulnois, Pinchard, Solère 199o. Je cite l'Elementatio theologica de Proclus dans l'édition de Dodds 1963, et dans la traduction de Trouillard 1965, avec quelques adaptations. 
dieux, les uns sous un mode unitaire, les autres sous un mode multiplié, les uns sous un mode sempiternel, les autres sous un mode temporel, les uns sous un mode incorporel, les autres sous un mode corporel.

Comme on peut le voir, l' auteur du De causis a intégralement repris le texte de Proclus tout en éliminant le sens polythéiste de l'argument. Mais, même avec cette modification, la proposition du De causis a déplu à Thomas d'Aquin. En effet, pour lui, il était inacceptable d'expliquer la diversité ontologique à partir de la réceptivité des êtres et non pas à partir de l'acte créateur lui-même. En effet, si les êtres reçoivent l' acte d'être différemment à cause de leurs puissances diverses, où faut-il chercher l' origine de cette différence dans leurs puissances? Thomas résout la difficulté en insistant sur le fait que l'auteur du $D e$ causis ne parle pas dans cette proposition de l'acte créateur de cette diversité, mais y explique comment le régime providentiel de la première cause s' étend à tous les êtres d'une manière différente:

Il faut prêter attention au fait que double est l' action de la cause première: par l'une, elle institue les choses, ce qu' on appelle création, par l' autre elle gouverne les choses déjà instituées ${ }^{3}$.

Ce dont parle l' auteur du De causis ne concerne donc pas l'acte de la création, dit Thomas, mais le gouvernement divin, car

la tout première diversité observée dans les choses selon qu' elles ont diverses natures et puissances, ne vient pas de la diversité des recevants mais de la cause première; et ce, non parce qu' il y aurait en elle quelque diversité, mais parce qu' elle connaît la diversité - car elle agit selon sa science. C' est pourquoi elle produit différents degrés de réalité pour achever l' univers ${ }^{4}$.

3 Thomas d'Aquin, Super Librum de causis expositio, 24, p. 122,19-21: Est autem attendendum quod duplex est actio causae primae: una quidem secundum quam instituit res, quae dicitur creatio, alia vero secundum quam res iam institutas regit. Je cite (avec des corrections) la traduction de Decossas 2005 .

4 Thomas d'Aquin, Super Librum de causis expositio, 24, p. 122,27-123,2: Unde oportet dicere quod prima diversitas rerum secundum quam habent diversas naturas et virtutes, non sit ex aliqua diversitate recipientium sed ex causa prima, non quia in ea sit aliqua diversitas sed quia est diversitatem cognoscens, est enim agens secundum suam scientiam; et ideo diversos rerum gradus producit ad complementum universi. 
Comme on le voit, Thomas comprend la création comme une création $e x$ nihilo à partir d' une cause qui agit selon la connaissance, c' est-à-dire, selon ses idées créatrices. L'auteur du De causis, en revanche, comprend la création plutôt comme une émanation et une diversification à partir du don de l'être qui vient de la première cause ${ }^{5}$. Il est donc inutile pour lui de distinguer entre un premier acte de création et un second acte de gouvernement. La diversité des êtres créés se manifeste dans les manières différentes dont ils procèdent de la bonté du Premier. Et cette différence entre les êtres s' exprime primordialement dans la distinction entre les êtres qui sont causes d' eux-mêmes et ceux qui dépendent d'une cause extérieure à eux-mêmes. Les êtres auto-constituants sont simples, incorporels, incorruptibles, sempiternels, les êtres causés par une autre cause sont composés, corporels, engendrés, corruptibles, temporels.

Après cette proposition générale XXIII(XXIV), qui sert d'introduction à tout l'exposé qui suit, l'auteur du De causis présente dans les propositions $\operatorname{xxIV(XXV)-XXVIII(XXIX)~la~classe~des~êtres~auto-constituants,~qui~ne~sont~pas~}$ soumis à la génération, non divisibles et hors de la temporalité, et il montre en quoi ils diffèrent des êtres engendrés, corruptibles, composés, temporels. La série des propositions correspond à l'Éléments de théologie de Proclus de la manière suivante:

Livre des causes, prop. $\operatorname{xxIV}(\mathrm{xXv})=\quad$ Éléments de théologie, $\S 45$ Livre des causes, prop. $\mathrm{xxv}(\mathrm{xxVI})=\quad$ Éléments de théologie, $\$ 46$ Livre des causes, prop. $\mathrm{XXVI}(\mathrm{XXVII})=$ Éléments de théologie, $\$ 48$ Livre des causes, prop. XXVII $(\mathrm{XXVII})=$ Éléments de théologie, $\S 47$ Livre des causes, prop. XXVIII $\left(\mathrm{XXIX)}=\right.$ Éléments de théologie, $\S_{51}$

On remarquera que dans le De causis la proposition qui correspond au $§ 48$ vient avant le correspondant du $\S 47$, ce qui n' est pas accidentel. Il me semble que l'auteur du De causis a voulu continuer après $\S 46$ avec $\S 48$ pour opposer immédiatement la classe des êtres engendrés à la classe des auto-constituants qui ne sont pas soumis à la génération. Après avoir montré cette distinction, il continue avec la démonstration de la simplicité des auto-constituants (\$ 47$)$. Il faut admettre que cette séquence est plus logique que ce que nous trouvons

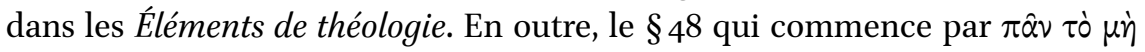

5 Sur la doctrine de la création dans le De causis et saint Thomas existe une ample littérature: cf. D’ Ancona 1995; Kremer 1966. 


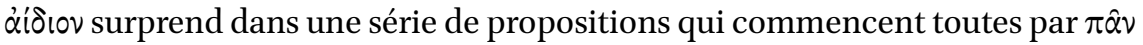
$\tau \grave{~} \alpha$ $\theta v \pi o ́ \sigma \tau \alpha \tau o v$. On a l'impression qu' une sentence fait défaut.

La dernière proposition sur les auto-constituants (Livre des causes, prop. XXVIII $($ XXIX $)$ Éléments de théologie \$51) sert, chez Proclus, de transition à la discussion suivante, c' est-à-dire les propositions sur le temps et l'éternité. En effet, comme le démontre Proclus au $§ 51$, les auto-constituants transcendent toute temporalité. Il faudra donc expliquer dans la suite comment le temporel et l'intemporel se distinguent. En suivant Proclus, l'auteur du De causis ne distingue pas simplement entre le temporel et l'éternel, mais il fait aussi une distinction, parmi les êtres temporels, entre ceux qui existent pour toujours et ceux qui ont leur existence dans une période limitée du temps (Livre des causes, prop. $\operatorname{XXIX}(\mathrm{XXX})=$ Éléments de théologie $\$ 55)$. Cette distinction le conduit à discuter ensuite l' état intermédiaire entre la véritable éternité, qui est au-delà de tout temps, et la temporalité, c' est-à-dire les êtres qui sont sempiternels (Livre des causes, prop. $\mathrm{XXX}(\mathrm{XXXI})-\mathrm{XXXI}(\mathrm{XXXII}))$, ce qui correspond chez Proclus aux §106-107. Finalement, l'auteur du De causis conclut son traité en exploitant dans la partie finale de la dernière proposition XXXI(XXXII) ce qu'il a trouvé chez Proclus dans la proposition 116: «Tout dieu est participable sauf l' Un ».

L'auteur du De causis a donc composé la dernière partie de son traité en sélectionnant intelligemment des propositions dans l'Elementatio de Proclus et en les adaptant à son intention. Cependant, on pourrait s' étonner du fait que les propositions 40 à 44 de Proclus ne trouvent pas d' équivalent dans le $D e$ causis. C'est surprenant parce que, dans ces propositions, Proclus démontre pour quelles raisons il faut admettre une classe d'êtres qui se constituent euxmêmes. Voici la liste de ses propositions dans la traduction de Trouillard:

40. Toutes choses qui procèdent d'autres causes sont subordonnées à celles qui se constituent par elles-mêmes et qui possèdent une essence auto-constitutive.

41. Tout ce qui est en un autre est produit seulement par un autre, alors que tout ce qui est en soi-même est auto-constitutif.

42. Tout ce qui est auto-constitutif a la capacité de se convertir vers soi.

43. Tout ce qui est capable de se convertir vers soi-même est autoconstitutif.

44. Tout ce qui est capable de se convertir vers soi selon l'activité est aussi converti vers soi selon l' essence.

Tandis que Proclus élabore soigneusement sa démonstration de la classe d'êtres auto-constituants, l' auteur arabe, en revanche, semble introduire cette classe abruptement, sans préparation argumentative. Cependant, si l' on consi- 
dère l' ensemble du De causis, on verra que l' auteur arabe a déjà démontré indirectement l' existence de cette classe d'auto-constituants plus haut dans son traité, dans la célèbre proposition $\mathrm{XIV}(\mathrm{xV})$ : «Tout être connaissant qui connaît sa propre essence revient à cette essence d'un retour total» («Omnis sciens qui scit essentiam suam est rediens ad essentiam suam redditione completa»). L' auteur y explique que ce «retour total» se réalise parce que le connaissant et le connu sont chose une dans l' acte de connaissance. Un tel retour total n' est possible que pour une substance qui est «stable, fixée en elle-même, sans avoir besoin dans sa fixation d'une autre chose pour l'établir, car elle est une substance simple et qui se suffit à soi» («quia est stans, fixa per se, non indigens in sui fixione et sui essentia re alia rigente ipsam, quoniam est substantia simplex, sufficiens per se ipsam»). Une telle substance correspond parfaitement à la description de l'auto-constituant, bien que l'auteur utilise ici un vocabulaire différent. Déjà Thomas avait constaté que l'auteur du De causis avait exploité dans la proposition $\mathrm{XIV}(\mathrm{XV})$ plusieurs propositions des Éléments de théologie de Proclus: $§ 83$ sur la connaissance de soi-même, §15-16 sur la réflexivité et finalement $\S 44-45$, c' est-à-dire les propositions qui démontrent qu'un être qui a la capacité de se convertir intégralement à lui-même dans l'acte de la connaissance est un être qui se constitue lui-même. L'auteur du De causis a donc bien compris l'importance capitale de cet argument pour établir l'existence de la classe des êtres auto-constituants dont il parlera à la fin de son traité.

Examinons maintenant de plus près les propositions $\operatorname{xxIV}(\mathrm{XXV})$-XXVIII (XXIX) qui traitent des auto-constituants en comparaison avec l'Elementatio theologica. Dans cette analyse je me laisse guider par Thomas d' Aquin, qui est le premier à avoir comparé minutieusement le De causis avec les Éléments de théologie de Proclus dont il avait reçu la traduction de Guillaume de Moerbeke ${ }^{6}$. Son commentaire montre qu' il a dû étudier intensivementl'Elementatio de Proclus, sinon il n' aurait pas pu trouver tant de parallèles (et de différences) entre ces deux textes. Car identifier les sources du De causis n' est pas chose facile, parce que l'auteur arabe ne suit jamais servilement le modèle dont il s'est inspiré, mais sélectionne des morceaux du texte proclien plus haut ou plus bas dans son propre texte.

Une remarque préliminaire concerne la traduction de $\alpha \dot{\theta} \theta 0 \pi \delta ́ \sigma \tau \alpha \tau o v$ par substantia stans per essentiam suam ou stans per seipsam, une substance qui subsiste par son essence, c' est-à-dire par elle-même, ce qui rend très bien le sens de

6 J'utilise à côté de l'édition de Saffrey 1954, la traduction et commentaire Decossas 2005 et la traduction annotée de D'Ancona 1986. Thomas a été le premier de profiter de cette nouvelle traduction, cf. Boese 1985 , p. 48-5o. 
l'expression grecque. Cependant, les commentateurs médiévaux se sentaient obligés d' expliquer la phrase substantia stans per essentiam suam. La formulation pose, en effet, des problèmes. Comme le dit Thomas, ce qui subsiste par son essence propre, c' est ce qui subsiste par soi-même; or subsister par soi-même est propre à chaque substance, qui se distingue par là des accidents. Faut-il donc admettre que chaque substance individuelle est un auto-constituant? Notons d' ailleurs que Philopon dans son commentaire sur les Catégories utilise le terme $\alpha \dot{\theta} \theta 0 \pi$ tó $\tau \alpha \tau$ ov pour chaque substance ${ }^{7}$. Thomas répond à cette objection, que l' auteur du De causis précise dans la proposition XXIV(xXv) dans quel sens il faut prendre stans per se ipsam en disant qu'il s' agit des substantiae unitae. Par cette addition - qu' on ne trouve pas dans le texte de Proclus l'auteur du De causis aurait voulu, dit Thomas, préciser qu'il s'agit dans la suite non pas des substances qui sont composées de matière et forme, mais de substances qui sont des formae per se existentes parce qu' elles ne sont pas reçues dans une matière, mais subsistent par elles-mêmes comme c' est le cas de l'intellect et des autres formes séparées ${ }^{8}$. En vrai néoplatonicien on pourrait même défendre que seules les substances incorporelles soient de véritables substances, tandis que ce qu' on appelle substances sensibles ne sont que des amalgames de matière et qualités, comme le pensait Plotin.

XXIV(XXv). Substantiae unitae intellectibiles non sunt generatae ex re alia.

Omnis substantia stans per essentiam suam est non generata ex re alia.

Quod si aliquis dicat possibile est ut sit generata ex re alia dicemus

si possibile est ut substantia stans per essentiam suam sit generata ex re alia procul dubio est substantia illa diminuta indigens

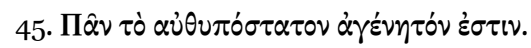

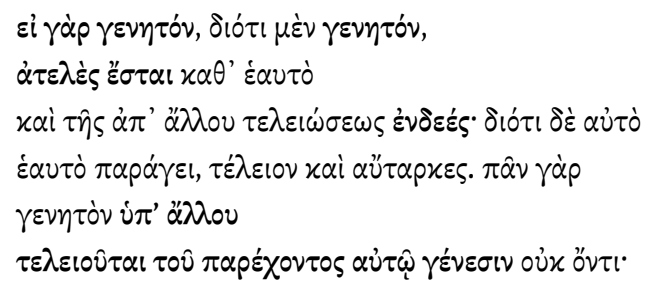

7 Philoponus, In Aristotelis Categorias commentarium 49.19-20 (cf. aussi 20.11, 46.16, 53.10): $\alpha$ $\tau \dot{\eta}$

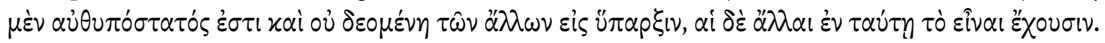

8 cf. Thomas d'Aquin, Super Librum de causis expositio, 25, p. 124,28-125,31; Gilles de Rome,

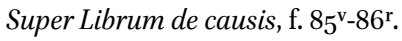


ut compleat eam illud ex quo generatur.

et significatio illius est generatio ipsa.

quod est quia generatio non est nisi via ex

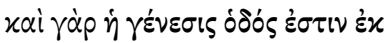

diminutione ad complementum.

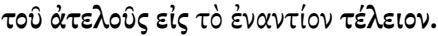

nam si invenitur res non indigens in genera-

tione sui scilicet in sua forma et sua forma-

tione re alia nisi se et est ipsa causa forma-

tionis suae et sui complementi et sui comple-

menti

est completa integra semper.

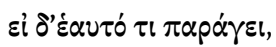

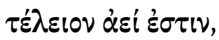

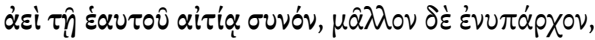

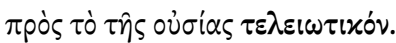

et non fit causa formationis suae et sui com-

plementi nisi propter relationem suam ad

causam suam semper. illa ergo comparatio est

formatio eius et ipsius complementum simul.

iam ergo manifestum est quod omnis substan-

tia stans per essentiam suam non est generata

ex re alia.

Comme on peut le voir dans ce tableau comparatif, l'auteur du De causis reste proche de l'argument de son modèle Proclus. Certes, le texte arabo-latin est plus développé que l'original grec. Ainsi, il a ajouté à la fin, en conclusion, quod erat demontrandum, ce que Proclus n' avait pas fait dans cette proposition: «iam ergo manifestum est quod omnis substantia stans per essentiam suam non est generata ex re alia». Mais plus surprenant est le fait que le chapitre commence par une double proposition: «Substantiae unitae intellectibiles non sunt generatae ex re alia. Omnis substantia stans per essentiam suam est non generata ex re alia». Thomas a bien remarqué que les deux propositions ne se trouvent pas dans la $\S 45$ de Proclus qui y correspond, mais seulement la seconde «Duarum autem propositarum propositionum prima in libro Procli non invenitur, sed solum secunda quae est XLVa sui libri». En plus, il note très judicieusement que l'auteur du De causis démontre dans la suite seulement la seconde proposition, s' accordant ainsi avec Proclus: «et haec sola propositio probatur consequenter eodem modo hic sicut et in libro Procli » ${ }^{9}$. Thomas

9 Thomas d'Aquin, Super Librum de causis expositio, 26, p. 125,16-20. 
aurait été heureux de savoir que sa perplexité sur la double forme de la proposition se reflète dans l'état du texte arabe tel qu'il nous a été transmis. En effet, la première proposition ne se trouve pas dans le manuscrit de Leyde, mais son copiste a noté dans la marge «j' ai trouvé écrit: Les êtres simples intelligibles ne sont pas engendrés d'autre chose» (cf. Bardenhewer, p. 104 note, et p. 8, p. 203, Pattin note l). Cependant, comme m'en a informé Cristina D'Ancona, dans le manuscrit d'Ankara on lit les deux propositions telles qu' elles se trouvent dans la version latine. Il me semble que le copiste du manuscrit de Leyde n'a pas eu l'intention de corriger son texte en indiquant dans la marge cette phrase à insérer. Il a simplement voulu dire qu' il trouvait cette formulation dans la marge de son modèle: «les êtres intelligibles etc.». Il est possible que l'auteur du De causis, quand il a commencé son exposé sur les êtres auto-constituants, ait noté dans la marge qu'il s' agissait des êtres intelligibles. Le manuscrit de Leyde représenterait ainsi l'état original, tandis que dans le manuscrit d'Ankara la remarque aurait été intégrée dans le texte. Quoi qu' il en soit, l' addition de substantiae unitae intellectibiles a marqué l'interprétation médiévale.

Examinons maintenant de plus près comment l'auteur du De causis a développé l'idée centrale de la proposition 45 des Éléments de théologie, c' est-à-dire l'explication de la manière dont ces substances sont causes d' elles-mêmes.

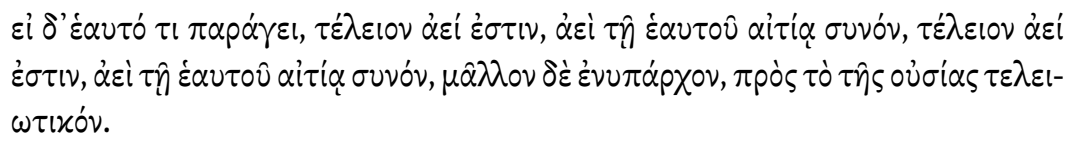

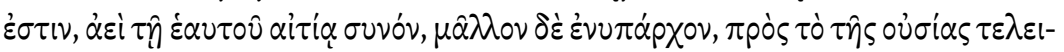

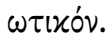

nam si invenitur res non indigens in generatione sui scilicet in sua forma et sua formatione re alia nisi se et est ipsa causa formationis suae et sui complementi, est completa integra semper, et non fit causa formationis suae nisi propter relationem suam ad causam suam semper.

Par cette élaboration du texte de Proclus, l'auteur du De causis précise en quel sens il faut comprendre ce que signifie être cause de soi-même. La prémisse de

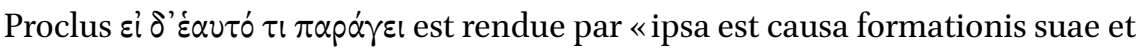
sui complementi»: une telle substance est «elle-même cause de sa propre formation et perfection ». Le terme «complementum» ne pose pas de problèmes:

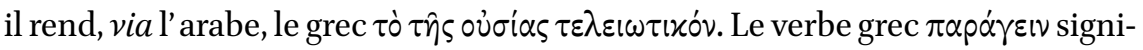
fie "produire, créer», mais l' auteur du De causis a préféré le terme formatio qu' il distingue de creatio, comme dans la célèbre proposition XVII(XVIII), qui établit une différence entre creatio, le don de l'être, qui dépend exclusivement de la causalité du premier principe, et formatio qui est la causalité de l'intellect 
et de la vie: «si ipsum dat omnibus rebus ens, tunc ipsum dat eis per modum creationis». La formation d'un être dans sa forme propre présuppose donc l'influx de l'être. Mais c' est la phrase suivante qui a surtout attiré l'attention des commentateurs latins: «et non fit causa formationis suae nisi propter relationem suam ad causam suam semper».

À propos de ce texte Thomas note: «mais afin qu' on ne comprenne pas à tort que ce type de substance n'a pas de cause à son être, l'auteur explicite, par la suite, comment ce qui est dit là doit être compris ${ }^{10}$. Par cette addition l' auteur aurait voulu éviter une interprétation fautive de sa thèse selon laquelle une telle substance est «cause de sa formation et de son achèvement». Il ne faut pas la comprendre comme si elle signifiait que cette substance ne dépendait pas de sa cause première. Comme l' explique Thomas, une telle substance est «cause de sa formation» du fait qu' elle a «une relation permanente à sa cause première ». Car, comme il s' agit de formes qui existent par elles-mêmes, leur rapport avec la cause première est perpétuel, ce qui n' est pas le cas des êtres composés de matière et forme, dont la matière est toujours en devenir et dont la forme advient avec leur génération et disparaît avec leur corruption. Ce n' est qu' au moment où advient la forme qu' un tel être est en relation avec sa cause première dont il reçoit l'être. Les formes qui existent par elles-mêmes, en revanche, ont toujours une relation avec leur cause première, ce qui explique pourquoi elles sont incorruptibles. Thomas fera la même remarque à propos de l'addition dans la proposition $\mathrm{xxv}(\mathrm{xxvI})$, dont je parlerai ultérieurement.

Gilles de Rome lui aussi explique que l' auteur du De causis a voulu, par cette addition, anticiper une objection sophistique («removet quandam cavillationem, vel respondet cuidam tacitae quaestioni (...)»). Quelqu'un aurait pu conclure du texte: «ergo talia non dependent ex causa prima». Et continuant l'argumentation de Thomas, Gilles explique que les formes qui subsistent par elles-mêmes n' ont pas besoin d' une autre cause « formaliter» (car comme elles sont elles-mêmes des formes, elles sont, en tant que telles, causes formelles de leur être); elles dépendent toutefois de la cause première comme de leur cause efficiente:

Res enim materiales indigent et alia causa formaliter et alia causa efficienter, sed res quae est ipsa forma, non indiget alia causa, nisi causa sua efficienter, non autem indiget alia causa formaliter ${ }^{11}$.

\footnotetext{
10 Thomas d'Aquin, Super Librum de causis expositio, 25, p. 126,9-12: ne ex hoc male intelligeret aliquis quod huiusmodi substantiae non haberent causam sui esse (...), manifestat consequenter quomodo sit intelligendum quod dictum est (traduction Decossas). 
Il faut toutefois remarquer que Thomas et tous les autres commentateurs latins lisent et interprètent le texte du De causis avec une addition («primam») qui ne se trouve nullement dans le texte latin et arabe, «nisi per relationem ad causam suam primam semper». C' est dans ce sens aussi qu'Albert le Grand avait déjà compris le texte:

Causa enim suae formationis et sui complementi non est nisi propter relationem ad causam suam priorem semper, hoc est, ut semper se referat ad causam primam se antecedentem. Refert autem se secundum suum esse. Secundum enim suum esse dependet ad causam priorem et hoc habet in seipsa $^{12}$.

Si l' on ne peut qu' admirer l'explication métaphysique qui est développée par la lecture de ce passage «nisi per relationem ad causam suam semper», il faut noter que ce n'est pas du tout ce que l'auteur du De causis a eu l'intention d'écrire. L' expression «relationem ad causam suam» est ambiguë. On pourrait la comprendre, comme le font les commentateurs latins, comme «une relation à sa cause », c' est-à-dire à la cause première. Mais cela n' est certainement

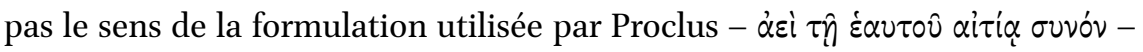
dont dépend le De causis. Proclus démontre par cela que la substance autoconstituante est incorruptible, «étant toujours conjointe à sa propre cause», et, comme il ajoute, «plutôt immanente» dans sa cause. La cause dont il s' agit n' est pas la cause première, mais l'essence ou l'être même de la substance qui se constitue elle-même. Il ne s'agit donc pas d'une relation avec une cause supérieure mais d'une connexion intime et indissoluble avec sa propre causalité. C'est parce qu' elles sont intimement liées à leur propre être qu'il faut exclure qu' elles soient générées ou produites. Par la phrase «nisi per relationem ad causam suam», l' auteur du De causis ne se distance donc nullement de Proclus, il ne veut nullement anticiper et résoudre un malentendu, il a sim-

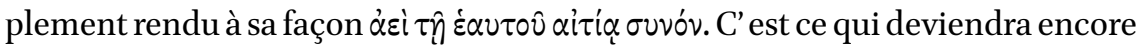
plus clair dans la proposition suivante.

12 Albert le Grand, De causis et processu universitatis a prima causa, lib. II, tr. 5, c. 5, p. 172,3035 ; cf. 171,65-67: Non enim habet causam suae formationis et esse nisi relationem suam ad causam primam, a qua accipit esse, 174,28: Formatio enim ipsarum relatio est ad causam primam. 
XXV(XXVI). omnis substantia stans per seipsam est non cadens sub corruptione.

si autem aliquis dicat possibile est ut substantia stans per seipsam cadat sub corruptione dicemus si possibile est ut substantia stans per seipsam cadat sub corruptione possibile est ut separetur eius essentia et sit fixa stans per essentiam suam sine essentia sua.

et hoc est inconveniens et impossibile quoniam propterea quod est una simplex non composita est ipsa causa et causatum simul. omnis autem cadentis sub corruptione non fit corruptio nisi propter separationem suam a causa sua.

dum vero permanet res pendens per causam suam retinentem eam et servantem eam non perit neque etiam destruitur.

si ergo hoc ita est

substantia stans ${ }^{13}$ per essentiam suam non separatur causa semper

quoniam est inseparabilis ab essentia sua

propterea quod causa eius est ipsa in formatione

sui.

et non fit causa sui ipsius nisi propter relationem suam ad causam suam et illa relatio est formatio eius.

et propterea quia est semper relata ad causam suam et ipsa est causa illius relationis est ipsa causa sui ipsius per modum quem diximus quod non perit neque corrumpitur neque etiam destruitur quoniam est causa et causatum simul sicut ostendimus nuper. iam ergo verificatum est quod omnis substantia stans per seipsam non destruitur neque corrumpitur.

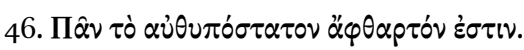

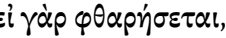

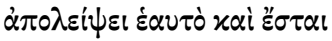

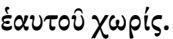

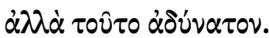

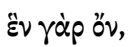

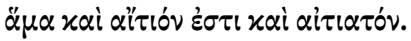

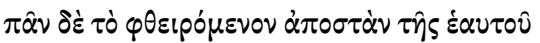

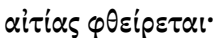

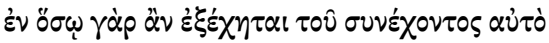

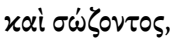

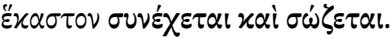

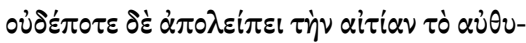
$\pi \dot{\sigma} \sigma \alpha \tau o \nu$,

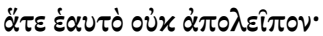

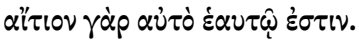

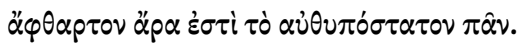

13 J'ai corrigé avec les manuscrits Paris. lat. 6318 et Vat. lat. 2089 le génitif «substantiae stan- 
Le texte central donne une nouvelle formulation de la thèse selon laquelle une substance auto-constituante n' est pas soumise à la génération et à la corruption, parce qu' elle est toujours liée à sa propre cause, étant elle-même la cause de son être.

si ergo hoc ita est substantia stans per essentiam suam non separatur causa semper quoniam est inseparabilis ab essentia sua propterea quod causa eius est ipsa in formatione sui.

Ce qui correspond au grec

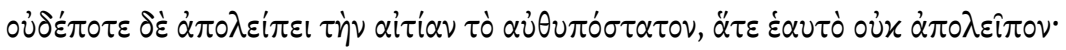

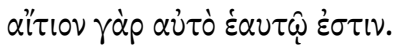

Mais l'auteur donne une explication qui ne se trouve pas chez Proclus:

Et elle ne devient cause de soi que par sa relation à sa cause et cette relation, c'est sa formation. Et pour cette raison, parce qu'elle est toujours reliée à sa cause, et qu' elle est elle-même cause de cette relation, elle est elle-même cause de soi-même, de la manière que nous avons indiquée: elle ne périt ni n' est détruite, puisqu' elle est à la fois cause et effet, comme nous l' avons démontré il y a peu.

Cette addition par rapport au texte de Proclus montre que l'auteur a absolument voulu expliquer en quel sens il faut comprendre que ces substances sont causes d'elles-mêmes. Faut-il donc dire avec Thomas qu' il a fait cette addition pour anticiper un malentendu?

afin qu'on n' aille toutefois pas croire que de telles substances ne dépendent pas d'une cause agente supérieure, l'auteur exclut ceci par la suite lorsqu' il dit: 'Et elle ne devient cause de soi etc.'14

tis» en «substantia stans», ce qui correspond au texte arabe que R. Taylor traduit ainsi: «Hence, if this is so, then the substance existing through its essence does not separate from its cause». La faute se trouvait probablement déjà dans l'archétype de toute la tradition latine.

14 Thomas d'Aquin, Super Librum de causis expositio, 26, p. 128,26-129,1: Sed ne aliquis credat quod huiusmodi substantiae stantes per essentiam suam non dependeant ab aliqua superiori causa agente, excludit hoc consequenter, ibi: et non est causa suipsius etc. (traduction Decossas). 
Je ne le crois pas. L' auteur reprend dans cette addition l' argument qu' il avait formulé dans la proposition précédente (per modum quam diximus), mais y ajoute l' explication qu'il vient de préciser dans la proposition actuelle (sicut ostendimus nuper), c'est-à-dire le fait que dans une telle substance la cause et l'effet de la cause ne font qu'un. C' est parce qu' elle est toujours reliée à la cause qu'elle est elle-même, qu' elle est cause d' elle-même («ipsa est causa sui ipsius»). On ne saurait donc, ici non plus, interpréter «causam suam» comme «causam suam primam». Dans cette proposition encore, l' auteur du De causis ne fait que développer ce que disait Proclus d'une manière succincte. Évidemment cela ne signifie pas que l'auteur du De causis exclut la dépendance des êtres auto-constituants de la cause première. D' ailleurs, Proclus lui-même ne le nie nullement. Mais ce n'est pas ce point que l'auteur arabe vise à établir dans cette proposition. Il s' agit seulement d' expliquer pourquoi ces êtres ne peuvent pas se corrompre: ils ne sont jamais séparés de leur propre cause, c' est-à-dire de leur être propre. Certes, les $\alpha \dot{\theta} \theta u \pi \delta ́ \sigma \tau \alpha \tau \alpha$ ne se créent pas, ne se génèrent pas, ils se constituent en donnant 'forme' à l' influx de l'être qui vient de la première cause, comme il est dit dans la proposition 18. Cette cause supérieure, que le De causis caractérise comme 'créatrice', n' est toutefois pas la cause efficiente de ces substances, comme le suggère Gilles de Rome.

On peut toutefois s'étonner du fait que Thomas n' ait pas critiqué l' auteur du De causis pour un autre problème. Si les substances auto-constituantes sont les causes formelles de leur propre être, elles existent toujours, de manière nécessaire, et ne peuvent pas être détruites (non perit neque etiam destruitur) ${ }^{15}$. Si Thomas ne fait pas d'objection à ce sujet, Gilles de Rome, en revanche, est très critique. Il croit même que l'argumentation dans cette proposition du De causis contient beaucoup de venin («multum venenum»). En effet, une substance qui subsiste par elle-même et est cause formelle de son être, est aussi cause et fondement (fundamentum) de sa relation à la cause première. Elle sera donc toujours conservée dans son être et existera nécessairement. Elle ne pourra jamais être annihilée, pas même par l' acte du créateur: «impossibile est ipsam omnino destrui vel adnihilari». Et Gilles note que, sur ce point, l' auteur du De causis est parfaitement d'accord avec Proclus: «Sic ergo sentiet iste Author, sic etiam sentit Proclus», «idem est ergo quod dicit Proclus et hic Author». Mais

15 Comme le disait Etienne Gilson (Gilson 1948, p. 93): «C'est un fait des plus inattendus, que l'univers de saint Thomas, effet contingent d' une volonté divine suprêmement libre, possède néanmoins la même stabilité et la même perdurabilité dans l'être que les mondes d'Aristote et d'Averroès ». Cf. D'Ancona 1986, p. 401 note. 
si c'est cela l' intention du texte, c' est un argument toxique qui risque de miner la croyance chrétienne d'un créateur suprêmement libre:

Diu autem loquuti sunt de intentione huius Authoris et Procli. Sed in his verbis maxime venenum latet. Nam voluerunt isti Philosophi quod substantiae immateriales essent simpliciter necesse esse et haberent necessitatem a seipsis ${ }^{16}$.

Même si ces êtres dépendent de la première cause effective, ils sont «causae suae necessitatis formaliter ». Mais ce qui formaliter est le fondement de la relation ne peut jamais perdre cette relation, sauf s'il se perdait lui-même et était séparé de lui-même, ce qui est impossible. Il se donne donc nécessairement son être en relation avec la première cause. Pour échapper à cette spirale empoisonnée (involutionibus) de raisonnements, il n'y a qu' une solution, pense Gilles. Il faut admettre que les intelligences (qui sont, selon lui, les substances autoconstituantes) ne sont pas absolument simples, mais composées elles-mêmes de forme et d'être, ou, dans le vocabulaire scolastique, d'essence et d'être («compositae ex essentia et esse vel ex forma et esse»). Dans cette hypothèse,

la relation que l'intelligence a avec la cause première comme sa cause efficiente n' est pas fondée sur la forme en tant que telle, ni sur son être en tant que tel, mais sur sa forme en tant qu' elle est perfectionnée par l' être et son être en tant qu'il perfectionne la forme. De cette manière la forme est l' effet du Premier, non pas que la forme soit produite en tant que telle, mais qu' elle soit l' effet du Premier pour autant que l' être lui est communiqué à partir du Premier ${ }^{17}$.

Tout comme les êtres dans le monde de la génération qui sont composés de matière et de forme peuvent se corrompre et périr, ainsi les intelligences elles aussi pourraient être détruites pervoluntatem primi. Car si une forme qui subsiste par soi, comme l' intelligence, n' est pas identique à son être, mais reçoit l'acte de l'être, elle peut être séparée de cet être et perdre ainsi sa relation avec la première cause. Gilles conclut que

\footnotetext{
16 Gilles de Rome, Super Librum de causis, f. $9 \mathrm{o}^{\mathrm{v}}$.

17 Gilles de Rome, Super Librum de causis, f. $91^{\mathrm{r}}$ : Relatio ergo quam habet intelligentia ad primam causam tanquam ad suum productivuum non fundatur in ipsa forma secundum se, nec in ipso esse secundum se, sed fundatur in forma ut perficitur per esse, et in esse ut perficit formam. Nam hoc modo forma est effectus primi: non quia ipsa producatur secundum se, sed est effectus primi prout a primo communicatur ei esse.
} 
les arguments de l'auteur du De causis et de Proclus ne concluent rien contre ceux qui, comme nous, admettent que, dans tous les êtres crées, l'être est réellement différent de l' essence; mais nous ne voyons pas comment ceux qui défendent que l' essence est réellement identique à l'être peuvent échapper à leur conclusion ${ }^{18}$.

En effet, si la destruction d'une forme signifie qu' elle est séparée de son être, et si son être ne peut pas être séparé d'elle-même, parce qu'il est identique à la forme, cette forme ne peut nullement être annihilée par la volonté de la première cause. Mais cela rendrait la création d'une telle forme nécessaire et ne ferait pas d' elle l' effet d' une volonté libre. C' est pour cette raison qu' il faut défendre qu'il existe une différence réelle entre la forme (ou l' essence) et l'être. Car, même si «selon l'ordre de la nature» (secundum naturae cursum) une intelligence est indissoluble, elle peut «par la volonté divine» (pervoluntatem primi) être annihilée.

\section{Livre des causes}

Proclus, Les Éléments de théologie

XXVI(XXVII). Omnis substantia destructibilis non

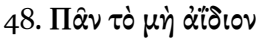

sempiterna

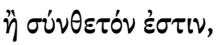

aut est composita

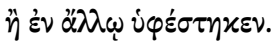

aut est delata super rem aliam.

propterea quod substantia

aut est indigens ${ }^{19}$ rebus ex quibus est

et est composita ex eis

aut est indigens in fixione sua et sua essentia deferente.

cum ergo separatur deferens eam corrumpitur et destruitur.

quod si substantia non est composita neque delata

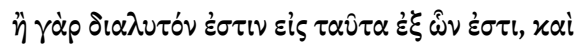

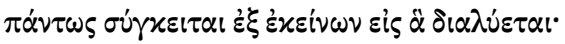

est simplex et semper non destruitur neque

minuitur omnino.

18 Gilles de Rome, Super Librum de causis, f. $9^{1}{ }^{\mathrm{r}}$ : contra nos ergo ponentes esse realiter differre ab essentia in omnibus creatis rationes huius Authoris et Procli non concludunt, sed non videmus quod non concludant contra ponentes essentiam idem esse realiter quod esse.

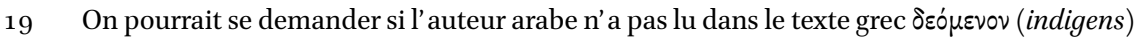
au lieu de $\delta\llcorner\alpha \lambda \cup \tau o ́ v$. Mais il peut également s' agir d'une adaptation. 
La proposition XXVI(XXVII) correspond parfaitement à la proposition 48 des Éléments de théologie ${ }^{20}$.

XXVII(XXVIII). Omnis substantia stans per essentiam suam est simplex et non dividitur.

Quod si dixerit aliquis possibile est ut dividatur dicemus

si possibile est ut substantia stans per se dividatur et est ipsa simplex

possibile est ut essentia partis eius sit per essentiam eius iterum

sicut essentia totius.

si ergo possibile est illud redit pars super seipsam et est omnis pars eius rediens super seipsam sicut est reditio totius super essentiam suam. et hoc est impossibile.

si ergo est impossibile

substantia stans per seipsam est indivisibilis et est simplex.

si autem non est simplex sed est composita pars eius est melior parte et pars eius vilior parte, ergo res melior est ex re viliori et res vilior ex re meliori quando est omnis pars eius seiuncta ab omni parte eius.

quare est universitas eius non sufficiens per seipsam cum indigeat partibus suis ex quibus componitur. et hoc quidem non est de natura rei simplicis immo de natura substantiarum compositarum.

iam ergo constat quod omnis substantia stans per essentiam suam est simplex non dividitur et quando non recipit divisionem et est simplex non est recipiens corruptionem neque destructionem.

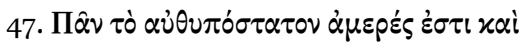
$\dot{\alpha} \pi \lambda \circ \hat{\nu}$.

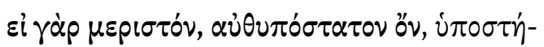

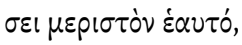

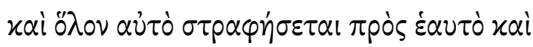
$\pi \hat{\alpha} \nu$ ह่ $\pi \alpha \nu \tau i$ ह่ $\alpha \nu \tau \hat{\omega}$

है $\sigma \tau$ เ.

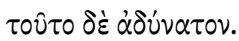

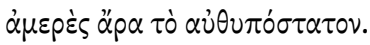

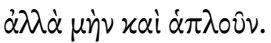

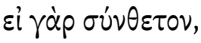

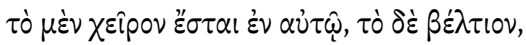

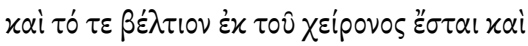

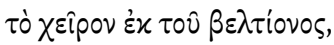

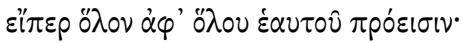

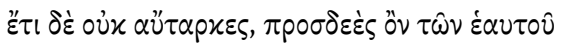

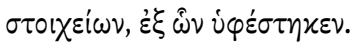

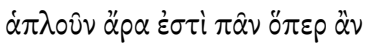

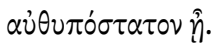

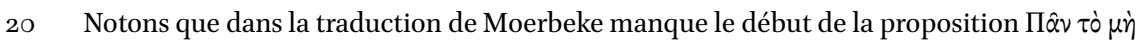
¿̇î̄ı 
La démonstration de la proposition 47 des Éléments pose plusieurs problèmes d' interprétation. Proclus démontre d' abord que l' auto-constituant est indivisible, ensuite qu'il est simple. Voici l'argument qui exclut la divisibilité:

Car s'il est divisible tout en étant auto-constituant, il se constituera luimême comme divisible, et cependant il se tournera dans sa totalité vers lui-même et sera tout entier en lui-même tout entier. Ce qui est impossible.

L' auteur du De causis interprète l' argument ainsi :

S'il est possible qu' une substance subsistant par elle-même soit divisible en même temps qu' elle est simple, alors il est possible que l'essence d'une de ses parties soit à son tour $\left\langle\right.$ subsistante $\left.{ }^{21}\right\rangle$ par son essence même, comme l'est l'essence du tout. Si cela donc est possible, cette partie fera retour sur elle-même, et chacune de ses parties fera retour sur elle-même, de même qu'il y a retour du tout sur sa propre essence. Or cela est impossible.

Je comprends l'argument de Proclus ainsi: si l'auto-constituant est divisible, il se constituera lui-même comme un être divisible, mais, puisqu'il est autoconstituant, il sera en même temps capable de se convertir vers lui-même dans sa totalité. Or, il a été démontré qu'un être divisible ne peut se convertir à lui-même intégralement (cf. §42-43). L'auteur du De causis comprend l'argument d' une autre façon: si l' auto-constituant est divisible, chacune de ses parties sera auto-constituante comme l' est le tout lui-même, ce qui est impossible. Thomas note que la version de l' argument dans le De causis est moins «efficace» que celui de Proclus, car, dit-il, «il n'est pas nécessaire que tout ce qui par soi convient au tout convienne aussi à chacune de ses parties». Et il croit que Proclus a mieux formulé la preuve qu'il présente ainsi :

Si un être divisible subsiste par lui-même, il faudrait que n' importe quelle partie subsiste par n'importe quelle autre et que n'importe quelle partie soit fondée en n'importe quelle autre. Ceci est impossible puisqu'il

tradition latine de l'Elementatio cette omission a été complée de manières diverses, parfois même en s' inspirant du De causis (comme dans le ms. Erfurt, Universitätsbibliothek, MS CA Amplon. $2^{\circ}$ 26), cf. Boese 1985 p. 29-30.

21 Stans ne se trouve pas dans le texte du De causis, mais est impliqué (cf. D'Ancona 1986, p. 408, n. 1), ou faut-il conjecturer «possibile est ut essentia partis eius stet per essentiam eius»? 
s' ensuivrait qu' une seule et même partie serait à la fois et au même point de vue cause et effet ${ }^{22}$.

Cependant, je ne crois pas que Thomas a mieux compris Proclus que l' auteur du De causis. Thomas semble avoir mal compris «totum ipsum vertertur ad seipsum et omne in omni seipso erit» comme «quaelibet fundatur in qualibet».

Pour démontrer que les auto-constituants sont des substances simples, Proclus utilise deux arguments. Le premier se retrouve exactement dans le De causis:

Proclus: Bien plus, il est simple. Car s'il était composé, il y a aura en lui du pire et du meilleur, et le meilleur viendra du pire et le pire du meilleur, puisqu' il procède tout entier de lui-même tout entier.

De causis: À supposer qu' elle ne soit pas simple, mais composée, alors une partie est meilleure que l' autre, une partie pire que l' autre, et le meilleur provient du pire et le pire du meilleur, puisque chacune des parties est séparée de chacune de ses autres parties.

L' argument de Proclus est surprenant. Pourquoi un être composé doit-il nécessairement comprendre une partie meilleure et une partie inférieure? C'est l'objection de Nicolas de Méthone ${ }^{23}$. Mais Dodds (p. 226) répond que Proclus suit ici Aristote qui, dans Métaphysique 1088b15, démontre que, dans un être composé, il y toujours une partie qui fonctionne comme 'matière'. Thomas argumente dans le même sens. Dans tout composé de parties multiples, dit-il, il y a un ordre dans les parties.

Aussi voyons-nous que dans la composition du corps naturel, la forme l'emporte sur la matière; dans celle des corps mixtes, un élément domine; dans la composition des parties de l'animal un membre est principal, etc $^{24}$.

Thomas d'Aquin, Super Librum de causis expositio, 28, p. 132.16-20: Si ergo aliquid partibile sit stans per seipsum, oportebit quod quaelibet pars eius stet per quamlibet et quaelibet fundetur in qualibet; quod est impossibile, quia sic sequeretur quod una et eadem pars eius esset causa et effectus simul respectu eiusdem, quod est impossibile. (traduction Decossas légèrement modifiée).

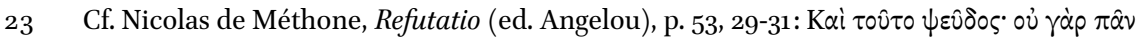

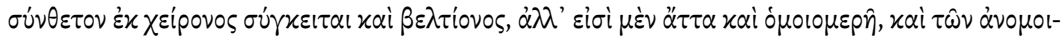

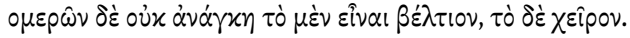


L' explication ne me convainc pas. Comment peut-on dire que «dans le composé le meilleur provient du pire et le pire du meilleur»? L'explication que donne Proclus est tout aussi obscure: "puisqu'il procède tout entier de luimême tout entier». L' auteur du De causis l' exprime l' argument ainsi: «puisque chacune des parties est séparée de chaque autres de ses parties». Thomas l'explique encore différemment: si ce qui est composé de plusieurs parties subsiste par soi-même, il faut que chaque partie subsiste par chaque partie et ainsi le meilleur par l'inférieur et l' inférieur par le meilleur. Il me semble que Proclus considère comme 'meilleure' la partie de l' auto-constituant qui constitue, et 'inférieure' la partie qui est constituée. Mais le texte reste énigmatique. Le second argument est plus clair: «en outre, il ne sera pas autosuffisant puisqu' il dépendra de parties dont il est composé », ce qui n'est pas possible pour une nature simple, comme l' ajoute le De causis. Pas de différence d' argument entre les deux versions, mais Thomas, qui a bien comparé les textes, observe que ce

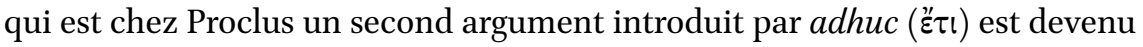
dans le De causis une conséquence: quare.

\section{Livre des causes}

XXVIII(XXIX). Omnis substantia [simplex est] stans per seipsam scilicet per essentiam suam [nam ipsa] est creata sine tempore et est in substantialitate sua superior substantiis temporalibus.

et significatio illius est quod non est generata ex aliquo quoniam est stans per essentiam suam

\section{Proclus, Éléments de théologie}

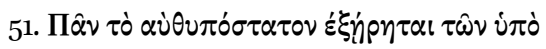

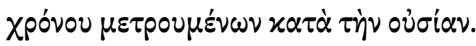

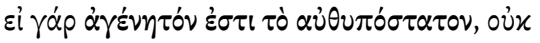

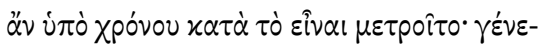

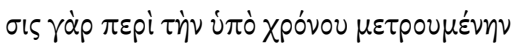

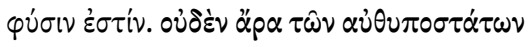

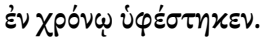

et substantiae generatae ex aliquo sunt substantiae compositae cadentes sub generatione.

iam ergo manifestum est quod omnis substantia stans per essentiam suam non est nisi in non tempore et qui est altior et superior tempore et rebus temporalibus.

compositione corporis naturalis forma est praestantior materia et in compositione corporis mixti unum elementum dominatur et in compositione partium animalium unum membrum principalius est alio (...) (traduction Decossas). 
Thomas fait remarquer que cette proposition «omnis substantia simplex est stans per seipsam» est la conversion («propositio conversa») de la précédente: «omnis stans per essentiam suam est simplex». Cependant, dit-il, ce n' est pas cette proposition qui est démontrée dans la suite, mais une autre proposition que l' auteur du De causis ajoute et qui est introduite par «nam», bien qu' elle ne donne pas la raison de ce qui précède: «Nam ipsa est creata sine tempore et est in substantialitate sua superior substantiis temporalibus». Ce qui suit est une démonstration de cette seconde proposition, et non pas de la proposition qui est mise en exergue. Ici encore, on voit comment Thomas a minutieusement comparé le De causis avec l'Elementatio de Proclus. Ce qu'il ne pouvait pas savoir, c' est que la confusion se trouve dans la version latine. En effet, dans l'original arabe les mots entre crochets [] manquent. Il n'y qu' une seule proposition qui se lit:

toute substance qui subsiste par elle-même, c' est-à-dire du fait de sa propre essence, est créée en dehors du temps, et dans sa propre substantialité au-dessus des substances temporelles

Ainsi la proposition correspond à Proclus, les Éléments de théologie §51. Il faut cependant noter l' addition dans De causis de «creata sine tempore» qui montre que ces substances ont reçu leur être de façon intemporelle.

\section{Conclusion}

Dans plusieurs endroits de son commentaire, Thomas montre qu'il apprécie davantage les arguments de Proclus que ce qu' il lit chez son imitateur, l' auteur du De causis: ils sont mieux formulés, plus clairs, plus efficaces dans la démonstration. D' autre part, il apprécie que l' auteur du De causis ait adapté et transformé le platonisme païen dans un sens qui le rend plus acceptable pour un chrétien: il a ainsi sa place à côté de Denys le Ps.-Aréopagite. Selon Thomas, une telle intervention se fait remarquer dans le commentaire des propositions $\mathrm{xxIV}(\mathrm{xxv})-\mathrm{XXv}(\mathrm{xxvI})$ à propos des êtres auto-constituants. L'auteur du De causis, dit Thomas, a voulu exclure une interprétation fautive de cette doctrine. Les êtres qui se constituent eux-mêmes ne peuvent le faire qu' en relation avec la première cause de tous les êtres. Comme je l'ai démontré, l'auteur du $D e$ causis reste plus proche de Proclus et sa doctrine de la procession des êtres que de la doctrine chrétienne de la création. Certes, il distingue la formatio de ces substances de leur creatio - une distinction qu' on ne trouve pas chez Proclus - mais sa conception de la création reste celle d'une émanation néces- 
saire. C'est le plus visible dans la doctrine des êtres constituants qui sont des êtres qui existent nécessairement, une doctrine que Thomas, malgré quelques réserves, adopte. Gilles de Rome a mieux remarqué le danger de la doctrine des $\alpha \dot{\theta} \theta 0 \pi \delta ́ \sigma \tau \alpha \tau \alpha$ pour la conception chrétienne d'un créateur libre. Et il a raison de dire que, sur ce point, il n'y a aucune différence entre Proclus et l' auteur du De causis.

\section{Remerciements}

Je remercie Alexandra Michalewski et Richard Taylor qui ont bien voulu relire mon texte.

\section{Bibliographie}

\section{Manuscrits}

Ankara, İsmail Saib I 1696

Erfurt, Universitätsbibliothek, MS CA Amplon. $2^{\circ} 26$

Leyde, Bibliotheek der Rijksuniversiteit, Oriental 209

Paris, Bibliothèque nationale de France, 6318

Vaticano (Città del), Vat. lat. 2089

\section{Sources primaires}

Albert le Grand, De causis et processu universitatis a prima causa II, 1, ed. W. Fauser, Münster, Aschendorff 1993 (Editio Coloniensis 17,2).

Gilles de Rome, Super Librum de causis, Venetiis, apud Iacobum Zoppinum, 155 o (repr. Frankfurt a.M. 1968).

Liber de causis: Die pseudo-aristotelische Schrift über das reine Gute bekannt unter dem Namen Liber de causis, ed. O. Bardenhewer, Freiburg im Breisgau, Herdersche Verlagschandlung, 1882 (reprint Frankfurt 1961); Pattin, A. "Le Liber de causis. Édition établie àl' aide de 90 manuscrits avec introduction et notes", in Tijdschriftvoor Filosofie 28, 1966, p. 9o-203 La demeure de l'être. Autour d'un anonyme. Etude et traduction $d u$ Liber de causis, trad. P. Magnard, O. Boulnois, B. Pinchard, J.-L. Solère, Paris, Vrin, 199o; Liber de causis. Das Buch der Ursachen, trad. A. Schönfeld, Hamburg, Meiner (Philosophische Bibliothek 553), 2003.

Nicholas of Methone, Refutation of Proclus' Elements of Theology. A critical edition with an introduction on Nicholas' life and works, ed. A.D. Angelou, Athens / Leiden, Academy of Athens / Brill, 1984.

Philoponus, In Aristotelis Categorias commentarium, ed. A. Busse, Berlin, Reimer, 1898. 
Proclus, The Elements of Theology. A Revised Text with Translation, Introduction and Commentary, ed. E.R. Dodds, Oxford, Clarendon Press, $1963^{2}$.

Proclus, Les Éléments de théologie, trad. J. Trouillard, Paris, Aubier-Montaigne, 1965.

Thomas d'Aquin, Super Librum de causis expositio, ed. H.-D. Saffrey, Fribourg, Société Philosophique, 1954.

Thomas d'Aquin, Commento al Libro delle cause, trad. C. D'Ancona, Milano, Rusconi, 1986.

Thomas d'Aquin, Commentaire du Livres des causes, trad. B. \& J. Decossas, Paris, Vrin, 2005 .

Thomas d'Aquin, Commentary on the Book of Causes, trad. V. Guagliardo, O.P., C. Hess, O.P., R.C. Taylor, Washington, D.C., Catholic University of America Press, 1996.

\section{Sources secondaires}

Beierwaltes, W. (2001), «Causa sui: Plotins Begriff des Einen als Ursprung des Gedankens der Selbstursächlichkeit», dans Das wahre Selbst. Studien zu Plotins Begriff des Geistes und des Einen, Frankfurt am Main, Vittorio Klostermann, p. 123-159.

Boese, H. (1985), Wilhelm von Moerbeke als Uebersetzer der Stoicheiosis theologike des Proclus, Heidelberg, Bitsch.

Chlup, R. (2012), Proclus: An Introduction, Cambridge, Cambridge University Press.

D’ Ancona, C. (1995), «La doctrine de la création mediante intelligentia dans le Liber de causis et ses sources», dans Ead., Recherches sur le Liber de causis, Paris, Vrin, p. 7395 .

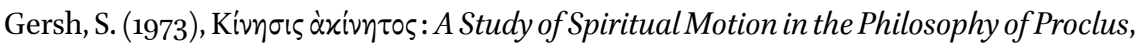
Leiden, Brill.

Gilson, E. (1948), L'être et l'essence, Paris, Vrin.

Kremer, K. (1966), Die neuplatonische Seinsphilosophie und ihre Wirkung auf Thomas von Aquin, Leiden, Brill.

Steel, C. (1998), «Conversion vers soi et constitution de soi selon Proclus», dans A. Charles-Saget (éd.), Retour, repentir et constitution de soi, Paris, Vrin, p. 161-175.

Steel, C. (2006), «Proklos über Selbstreflexion und Selbstbegründung», dans M. Perkams, R.M. Piccione (eds), Proklos. Methode, Seelenlehre, Metaphysik. Leiden / Boston, Brill, p. 230-255.

Taylor, R.C. (1989), « Remarks on the Latin Text and the Translator of the Kaläm fimahd al-khair / Liber de causis», dans Bulletin de philosophie médiévale 31, p. 75-102.

Trouillard, J. (1976), «Théologie négative et autoconstitution psychique chez les néoplatoniciens », dans Savoir, faire, espérer: Les limites de la raison. Mélanges Henrivan Camp, vol. I, Bruxelles, Presses de l'Université Saint-Louis, p. 307-321.

Whittaker, J. (1975), «The Historical Background of Proclus' Doctrine of the authypostata», dans H. Dörrie (éd.), De Jamblique à Proclus, Genève, Vandœuvres, p. 193-23o. Rep. in Studies in Platonism and Patristic Thought XVI, London, Aldershot, 1984. 